\title{
Channel and Delay Estimation Algorithm for Asynchronous Cooperative Diversity
}

\author{
Kamel TOURKI and Luc DENEIRE \\ Laboratoire I3S \\ 2000 route des Lucioles, B.P. 121, 06903 Sophia Antipolis Cedex, FRANCE. \\ Email: \{tourki, deneire\}@i3s.unice.fr
}

\begin{abstract}
For mobile users without antenna arrays, transmission diversity can be achieved with cooperative spacetime encoded transmissions. This paper present a channel and delay estimation algorithm for asynchronous cooperative diversity in Rayleigh Block-Flat-Fading channel. We present a precoding frame-based scheme with packet-wise encoding. This precoding is based on the addition of a cyclic prefix which is implemented as a training sequence. This scheme offers us additional known symbols and we show that it enables best synchronization and channel estimation which reaches the Cramer-Rao Bound. We derive the performance analysis for equal and unequal channel gains. The BER performances corroborate with the analytical expressions and the full diversity order is achieved. In addition, the FER performances are depicted.
\end{abstract}

Key words : Cooperative Diversity, Synchronization, Distributed Space-Time Coding, Cyclic Prefix, Rayleigh Block-FlatFading Channel.

\section{INTRODUCTION}

Multiple antennas at the receiver and the transmitter are often used to combat the effects of fading in wireless communication system. However, implementing multiple antennas at the mobile stations is impractical for most wireless applications due to the limited size of the mobile unit. So, active users pool their resources to form a virtual antenna array (VAA) that realizes spatial diversity gain in a distributed fashion. It is the cooperative diversity (CD) system. Space-Time Bloc Codes (STBC) has been naturally employed for improved bandwidth efficiency besides the targeted diversity benefits [1], [2] and [3]. Unfortunately, it is difficult, and in most cases impossible, to achieve perfect synchronization among distributed transmitters. Therefore a challenge is the lack of perfect synchronization on delay and mobility of distributed transmitters. This scheme is based on [4] and [5], by proposing a precoding based on the addition of a cyclic prefix which is implemented as a training sequence. In the system considered, the data is transmitted in packets via two cooperative mobiles and the channel is considered Rayleigh block-flat-fading. The placement of training sequence, concentrated toward the edges of the date packet [4], is optimal for asynchronous interference [6].

In section 2 the system model is discussed and simple mathematical descriptions are given. In section 3 , the channel and delay estimation algorithm, the detection schemes are discussed. In section 4 is for the simulation results, and we conclude by section 5 .

All boldface letters indicate vectors (lower case) or matrices (upper case). The $\operatorname{tr}(\mathbf{A})$ is the trace of matrix $\mathbf{A},()^{*},()^{H}$ and ()$^{\sharp}$ are the conjugate, the hermitian and the pseudo-inverse operators respectively. $\mathrm{E}\left[\mathrm{]}\right.$ is the Expectation operator, $\mathbf{I}_{N}$ is the identity matrix and $\mathbf{I}_{N}(L)$ is a matrix contains the $L$ latest rows on $I_{N} . \mathbf{O}_{N}$ is an $N \times N$ matrix with all elements equal to 0 , and we use $\mathbf{o}_{N}$ to denote an $N \times 1$ vector with all elements equal to $0 . \mathbf{a}(n)$ is the $n^{\text {th }}$ block symbols, and $\mathbf{a}(n, k)$ is the $k^{t h}$ element of $\mathbf{a}(n)$. The Complex Gaussian distribution with mean $\mu$ and covariance matrix $\mathbf{C}$ is denoted by $\mathcal{C N}(\mu, \mathbf{C})$.

\section{SYSTEM MODEL}

This paper focuses on the design of a channel and delay estimation algorithm for an asynchronous cooperative diversity system using a linear block precoding with a training sequence as cyclic prefix. The system considered has two transmit and one or two receive antennas. On a virtual MIMO link, multiple transmitters will transmit the same symbols to a common destination; this joint transmission improves the signal quality and therefore, the reliability of received information at the destination node. The symbols are replicated in space and time in a specific manner that enables the destination node to combine the received symbols in a simple manner (linear) to reap the benefits of diversity. The main principle underlying this block transmission system is that the block of symbols to be transmitted, instead of being sent directly, is parsed into two sub-blocks of $N$ symbols, $\mathbf{d}(n)$ and $\mathbf{d}(n+1)$, adding the training sequences $\mathbf{d}_{1}$ and $\mathbf{d}_{2}$ in each trail of the sub-blocks respectively, it can be seen in figure 1 . We obtain two $(N+L) \times 1$ vectors $\mathbf{s}(n)$ and $\mathbf{s}(n+1)$, which

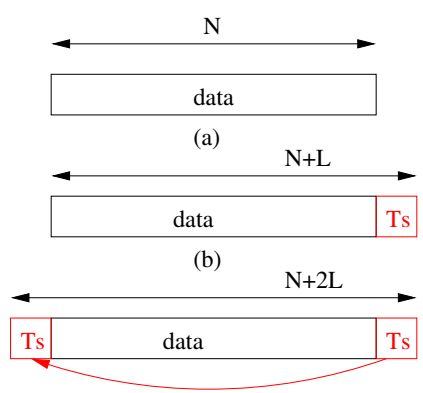

(c)

Figure. 1. A Frame description.

are represented by (b) in figure 1 . These vectors are represented in the relations 1 and 2 . We use the time reversal matrices $\mathbf{T}$ and $\mathbf{T}_{s}$ as linear precoding to obtain $\mathbf{s}_{v}(n)$ and $\mathbf{s}_{v}(n+1)$, which are 
Table 1. Transmission scheme

\begin{tabular}{|c|c|c|}
\hline & $n^{t h}$ block symbols & $(n+1)^{t h}$ block symbols \\
\hline ant $\mathbf{t} \mathbf{x}_{1}$ & $\mathbf{F}_{P} \times \mathbf{s}(n)$ & $-\left(\mathbf{F}_{P} \times \mathbf{s}_{v}(n+1)\right)^{*}$ \\
ant $\mathbf{t x}_{2}$ & $\mathbf{F}_{P} \times \mathbf{s}(n+1)$ & $\left(\mathbf{F}_{P} \times \mathbf{s}_{v}(n)\right)^{*}$ \\
\hline
\end{tabular}

represented in the relations 3 and 4 .

$$
\begin{aligned}
\mathbf{s}(n) & =\left[\begin{array}{c}
\mathbf{d}(n) \\
\mathbf{d}_{1}
\end{array}\right] \\
\mathbf{s}(n+1) & =\left[\begin{array}{c}
\mathbf{d}(n+1) \\
\mathbf{d}_{2}
\end{array}\right] \\
\mathbf{s}_{v}(n) & =\left[\begin{array}{c}
\mathbf{T d}(n) \\
\mathbf{T}_{s} \mathbf{d}_{1}
\end{array}\right] \\
\mathbf{s}_{v}(n+1) & =\left[\begin{array}{c}
\mathbf{T d}(n+1) \\
\mathbf{T}_{s} \mathbf{d}_{2}
\end{array}\right]
\end{aligned}
$$

Our method consists in inserting, between any two successive blocks, a cyclic prefix as it can be seen in (c) in figure 1. This operation is done when pre-multiplying in the left by $\mathbf{F}_{p}$, then $\mathbf{s}(n)$ and $\mathbf{s}_{v}(n)$ are extended to $(N+2 L)$ symbols. A distributed space time coding gives the transmitted frames $\mathbf{s}_{1}[n]$ and $\mathbf{s}_{2}[n]$ which are formed as in relations 5 and 6 . The transmission scheme is represented in the Table 1.

The precoding matrices $\mathbf{F}_{p}=\left[\begin{array}{c}I_{N+L}(L) \\ I_{N+L}\end{array}\right], \mathbf{T}$ and $\mathbf{T}_{s}$ are represented in figure 2.

$$
\begin{gathered}
\mathbf{s}_{1}[n]=\left[\begin{array}{c}
\mathbf{F}_{p} \mathbf{s}(n) \\
-\left(\mathbf{F}_{p} \mathbf{s}_{v}(n+1)\right)^{*}
\end{array}\right] \\
\mathbf{s}_{2}[n]=\left[\begin{array}{c}
\mathbf{F}_{p} \mathbf{s}(n+1) \\
\left(\mathbf{F}_{p} \mathbf{s}_{v}(n)\right)^{*}
\end{array}\right]
\end{gathered}
$$

Each link of this system is considered a point-to-point one
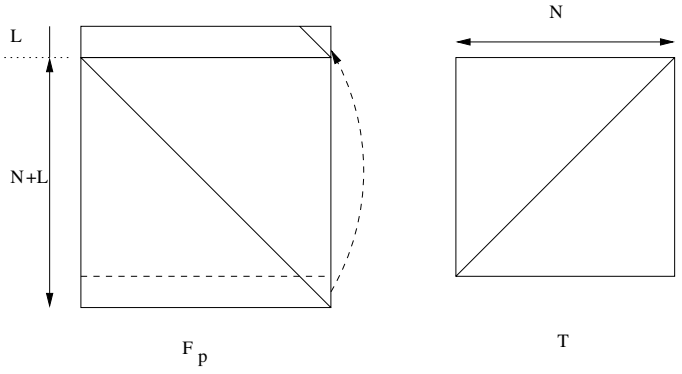

Figure. 2. The precoding matrices

way communication link, and the channel is assumed Rayleigh block-flat-fading. The mobile target receives a summation of the signals of the two active mobiles after they travel through different paths in the channel. These channel paths induce different delays, attenuations and phase shifts to the signals cause fading in the channel. Therefore, the transmission delays and channels can be estimated efficiently from training sequences. We define $\tau_{1}$ and $\tau_{2}$ respectively as the arrival time of the first and the second signals. We assume without loss of generality that $\tau_{1} \leq \tau_{2}$.

The received signal is given by

$$
\mathbf{r}=\mathcal{A}(\tau) \mathbf{X h}+\mathbf{b}
$$

where the total noise vector $\mathbf{b} \sim \mathcal{C N}\left(\mathbf{0}, N_{0} \mathbf{I}_{2 N+4 L}\right)$ and

$$
\begin{aligned}
& \mathcal{A}(\tau)=\left[\begin{array}{llll}
\mathbf{O}_{2 N+4 L} & \mathbf{I}_{2 N+4 L} & \Gamma & \Psi
\end{array}\right] \\
& \mathbf{X}=\left[\begin{array}{cc}
\mathbf{s}_{1}[n-1] & \mathbf{o}_{2 N+4 L} \\
\mathbf{s}_{1}[n] & \mathbf{o}_{2 N+4 L} \\
\mathbf{o}_{2 N+4 L} & \mathbf{s}_{2}[n-1] \\
\mathbf{o}_{2 N+4 L} & \mathbf{s}_{2}[n]
\end{array}\right] \\
& \mathbf{h}=\left[\begin{array}{l}
h_{1} \\
h_{2}
\end{array}\right]
\end{aligned}
$$

In the equation above $h_{1,2} \sim \mathcal{C N}(0,1)$ are the complex scalar channel parameters, $\mathbf{X}$ is the matrix obtained by stacking two consecutive frames from each transmitter. $\Gamma$ and $\Psi$ with size $(2 N+4 L) \times(2 N+4 L)$ account for the asynchronism between the two signals, and are expressed respectively as

$$
\begin{gathered}
\Gamma=\left[\begin{array}{cc}
\mathbf{O}_{L_{\tau} \times\left(2 N+4 L-L_{\tau}\right)} & \mathbf{I}_{L_{\tau}} \\
\mathbf{O}_{\left(2 N+4 L-L_{\tau}\right) \times\left(2 N+4 L-L_{\tau}\right)} & \mathbf{O}_{\left(2 N+4 L-L_{\tau}\right) \times\left(L_{\tau}\right)}
\end{array}\right] \\
\Psi=\left[\begin{array}{cc}
\mathbf{O}_{L_{\tau} \times\left(2 N+4 L-L_{\tau}\right)} & \mathbf{O}_{L_{\tau}} \\
\mathbf{I}_{2 N+4 L-L_{\tau}} & \mathbf{O}_{\left(2 N+4 L-L_{\tau}\right) \times\left(L_{\tau}\right)}
\end{array}\right]
\end{gathered}
$$

where $L_{\tau}=\tau_{2}-\tau_{1}$ is the relative delay which is bounded by $L$. We can remark that in synchronous case, $\Gamma_{\text {syn }}=\mathbf{O}_{2 N+4 L}$ and $\Psi_{\text {syn }}=\mathbf{I}_{2 N+4 L}$.

\section{CHANNEL AND DELAY ESTIMATION ALGORITHM}

A maximum likelihood (ML) method for delay and channel estimation is proposed. We denote $\mathbf{t s}_{\mathbf{1}}$ and $\mathbf{t s}_{\mathbf{2}}$ as

$$
\begin{aligned}
& \mathbf{t s}_{\mathbf{1}}=\mathbf{T}_{s} \mathbf{d}_{1} \\
& \mathbf{t s}_{\mathbf{2}}=\mathbf{T}_{s} \mathbf{d}_{2}
\end{aligned}
$$

Therefore we define $\mathbf{S}(\tau)=\left[\mathbf{s s}_{\mathbf{1}} \mathbf{s s}_{\mathbf{2}}\right]$ where

$$
\begin{gathered}
\mathbf{s s}_{\mathbf{1}}=\left[\begin{array}{c}
\mathbf{d}_{1}(\tau+1: L) \\
-\left(\mathbf{t s}_{\mathbf{2}}\right)^{*}
\end{array}\right] \\
\mathbf{s s}_{\mathbf{2}}=\left[\begin{array}{c}
\mathbf{d}_{2} \\
\left(\mathbf{t s}_{\mathbf{1}}(1: L-\tau)\right)^{*}
\end{array}\right]
\end{gathered}
$$

For this deterministic model, we denote $\mathbf{z}(\tau)=\mathbf{r}[n, N+L+$ $\tau+1: N+3 L]$, therefore we can write

$$
\widetilde{\mathbf{h}}(\tau)=(\mathbf{S}(\tau))^{\sharp} \mathbf{z}(\tau)
$$

then

$$
\widehat{\tau}=\arg \min _{\tau<L}\|\mathbf{z}-\mathbf{S}(\tau) \widetilde{\mathbf{h}}(\tau)\|^{2}
$$

and

$$
\widehat{\mathbf{h}}=(\mathbf{S}(\widehat{\tau}))^{\sharp} \mathbf{z}(\widehat{\tau})
$$

The Mean Square Error (MSE) of the channel estimation is defined by

$$
M S E(h)=\mathrm{E}\left[\|\mathbf{h}-\widehat{\mathbf{h}}\|^{2}\right]
$$

We studied the Cramer-Rao Bound for this model, we derive (see Appendix 6.1)

$$
C R B=N_{0}\left[(\mathbf{S}(\tau))^{H} \mathbf{S}(\tau)\right]^{-1}
$$




\subsection{Detection scheme for one receiver}

We denote $\mathbf{r}_{a}, \mathbf{r}_{b}$ and $\mathbf{y}$ as

$$
\begin{gathered}
\mathbf{r}_{a}=\mathbf{r}[n, L+1: N+L+\tau] \\
\mathbf{r}_{b}=\mathbf{r}[n, N+3 L+1: 2 N+3 L+\tau] \\
\mathbf{y}=\left[\mathbf{r}_{a}^{T} \mathbf{r}_{b}^{T}\right]
\end{gathered}
$$

The combiner builds the following two combined signals that are sent to the maximum likelihood detector:

$$
\begin{aligned}
\widetilde{\mathbf{d}}(n, k) & =\widehat{\mathbf{h}}_{1}^{*}(n) \mathbf{y}(n, k)+\widehat{\mathbf{h}}_{2}(n) \mathbf{y}^{*}(n, l+1-k) \\
\widetilde{\mathbf{d}}(n+1, k) & =\widehat{\mathbf{h}}_{2}^{*}(n) \mathbf{y}(n, \tau+k)-\widehat{\mathbf{h}}_{1}(n) \mathbf{y}^{*}(n, l-\tau+1-k)
\end{aligned}
$$

Where $l$ is the number of bits which $\mathbf{y}$ contains.

\subsection{Detection scheme for two cooperative receivers}

We receive $\mathbf{r}_{1}$ and $\mathbf{r}_{2}$ and we denote $\mathbf{r}_{1 a}, \mathbf{r}_{1 b}$ and $\mathbf{y}_{1}$ as

$$
\begin{gathered}
\mathbf{r}_{1 a}=\mathbf{r}_{1}\left[n, L+1: N+L+\tau_{1}\right] \\
\mathbf{r}_{1 b}=\mathbf{r}_{1}\left[n, N+3 L+1: 2 N+3 L+\tau_{1}\right] \\
\mathbf{y}_{1}=\left[\mathbf{r}_{1 a}^{T} \mathbf{r}_{1 b}^{T}\right]
\end{gathered}
$$

And we denote $\mathbf{r}_{2 a}, \mathbf{r}_{2 b}$ and $\mathbf{y}_{2}$ as

$$
\begin{gathered}
\mathbf{r}_{2 a}=\mathbf{r}_{2}\left[n, L+1: N+L+\tau_{2}\right] \\
\mathbf{r}_{2 b}=\mathbf{r}_{2}\left[n, N+3 L+1: 2 N+3 L+\tau_{2}\right] \\
\mathbf{y}_{2}=\left[\mathbf{r}_{2 a}^{T} \mathbf{r}_{2 b}^{T}\right]
\end{gathered}
$$

The combiner builds the following two combined signals that are sent to the maximum likelihood detector :

$$
\begin{aligned}
\widetilde{\mathbf{d}}(n, k)= & \widehat{\mathbf{h}}_{1}^{*}(n) \mathbf{y}_{1}(n, k)+\widehat{\mathbf{h}}_{2}(n) \mathbf{y}_{1}^{*}\left(n, l_{1}+1-k\right) \\
+ & \widehat{\mathbf{h}}_{3}^{*}(n) \mathbf{y}_{2}(n, k)+\widehat{\mathbf{h}}_{4}(n) \mathbf{y}_{2}^{*}\left(n, l_{2}+1-k\right)(33) \\
\widetilde{\mathbf{d}}(n+1, k)= & \widehat{\mathbf{h}}_{2}^{*}(n) \mathbf{y}_{1}\left(n, \tau_{1}+k\right)-\widehat{\mathbf{h}}_{1}(n) \mathbf{y}_{1}^{*}\left(n, \nu_{1}\right) \\
& +\widehat{\mathbf{h}}_{4}^{*}(n) \mathbf{y}_{2}\left(n, \tau_{2}+k\right)-\widehat{\mathbf{h}}_{3}(n) \mathbf{y}_{2}^{*}\left(n, \nu_{2}\right)(34)
\end{aligned}
$$

Where $l_{1}$ and $l_{2}$ are the numbers of bits which $\mathbf{y}_{1}$ and $\mathbf{y}_{2}$ contain respectively, and $\nu_{i}=l_{i}-\tau_{i}+1-k$.

\section{SIMULATIONS}

Each frame contains 288 symbols in which 224 for data. Therefore the cyclic prefix contain $(2 \times 16)$ symbols for training sequence. For burst transmission, each burst contain 100 frames. Figure 3 shows the MSE of the channel estimation performance, for which we derive $\operatorname{tr}(C R B)$ for comparisons. We can remark that the $\mathrm{CRB}$ is reached. We derive the performance for constant delay (A) and burst transmission (B) schemes.

For equal sub-channel gains $\gamma$, the moment generating function (MGF) of the instantaneously experienced SNR for a system with $t$ transmit antennas, $r$ receive antennas and $\lambda$ is the channel energy, can be expressed as

$$
\phi_{\frac{1}{R} \frac{\lambda}{t} \frac{S}{N}}(s)=\frac{1}{\left(1-\frac{1}{R} \frac{\gamma}{t} \frac{S}{N} \times s\right)^{u}}
$$

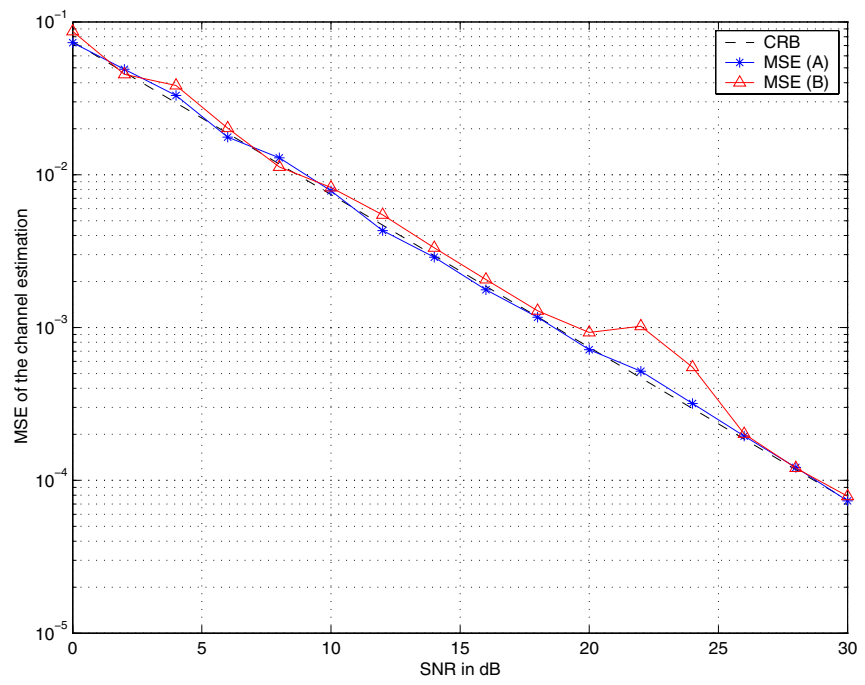

Figure. 3. The performance of the channel estimation.

where $R$ is the transmission rate and $u=t \times r$. The analysis in [7] allows expressing the BER of BPSK in closed form as

$$
\begin{aligned}
P_{t, r}(e)= & \phi_{\frac{1}{R} \frac{\lambda}{t} \frac{S}{N}}(-1)\left[\frac{1}{2 \sqrt{\pi}} \frac{\Gamma(u+1 / 2)}{\Gamma(u+1)}\right] \times \\
& { }_{2} F_{1}\left(u, 1 / 2 ; u+1 ;\left(1+\frac{1}{R} \frac{\gamma}{t} \frac{S}{N}\right)^{-1}\right)
\end{aligned}
$$

where ${ }_{2} F_{1}(a, b ; c ; x)$ is the Gauss hypergeometric function with 2 parameters of type 1 and 1 parameter of type 2 . It has been implemented using the series representations

$$
{ }_{2} F_{1}(a, b ; c ; x)=\sum_{n=0}^{\infty} \frac{(a)_{n}(b)_{n}}{(c)_{n} n !} x^{n}
$$

where

$$
(a)_{n}=\frac{\Gamma(a+n)}{\Gamma(a)}
$$

We can note $P_{t, r}(e)$ as $P s e\left(R, \gamma, \frac{S}{N}, t, r\right)$.

For unequal sub-channel gains, the MGF is shown to be

$$
\phi_{\frac{1}{R} \frac{\lambda}{t} \frac{S}{N}}(s)=\sum_{i=1}^{u} K_{i} \phi_{\frac{1}{R} \frac{\lambda_{i}}{t} \frac{S}{N}}(s)
$$

with constants $K_{i}$ (see Appendix 6.2)

$$
K_{i}=\prod_{i^{\prime}=1, i^{\prime} \neq i}^{u} \frac{\gamma_{i}}{\gamma_{i}-\gamma_{i^{\prime}}}
$$

where $\gamma_{i}$ is the average channel gain of the $i^{\text {th }}$ path. This allows one to derive the closed form BER where all the channel gains differ. The error rate can be expressed as

$$
P_{u c g}(e)=\sum_{i=1}^{u} K_{i} P \operatorname{se}\left(R, \gamma_{i}, \frac{S}{N}, 1,1\right)
$$

Explicitly, figure 4 depicts the BER versus the SNR in [dB] for distributed Alamouti scheme with one receive antenna. The 


\section{WPMC 2006 - San Diego, CA, USA}

power of the unequal channel coefficients is chosen such that $\gamma_{1}+\gamma_{2}=2$. Clearly, the distributed scenario provides the diversity gain even for the case of unbalanced channel gains. These are illustrated in figure 4 and figure 6 where analytical expressions corroborate with numerical simulations. Figure 5

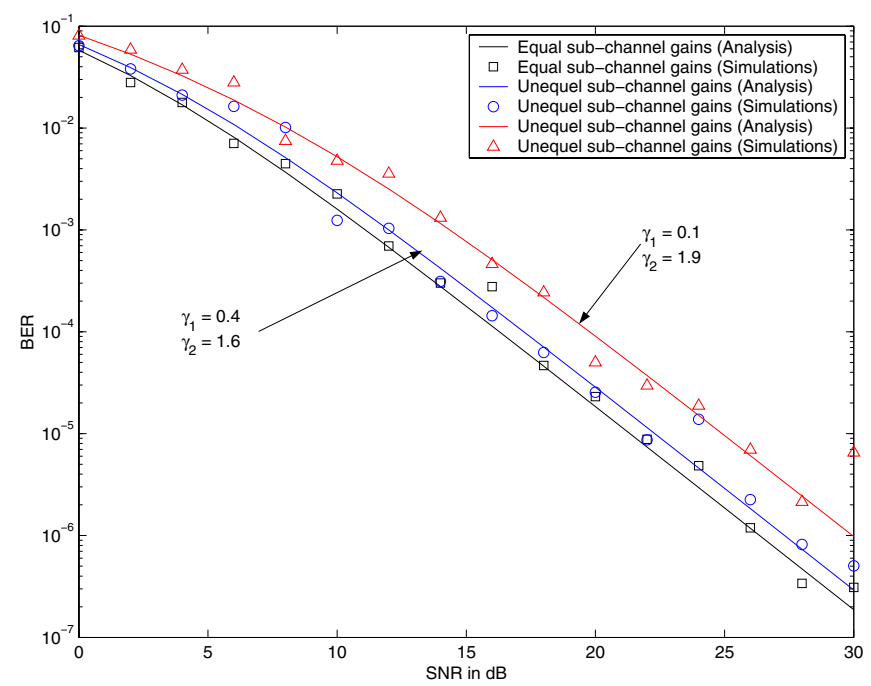

Figure. 4. BER performances of the asynchronous cooperative diversity system with channel and delay estimations for one receiver case, with equal and unequal sub-channel gains.

depicts the BER versus the normalized power $\gamma_{1}$ in the first link for the distributed Alamouti scheme with an SNR of $30 \mathrm{~dB}$. We remark that, in figure 4 , the BER performances attain respectively $10^{-6}$ and $3 \times 10^{-7}$ when $\gamma_{1}=0.1$ and $\gamma_{1}=0.4$ at SNR of $30 \mathrm{~dB}$. As showed in figure 7, the frame-error rate (FER)

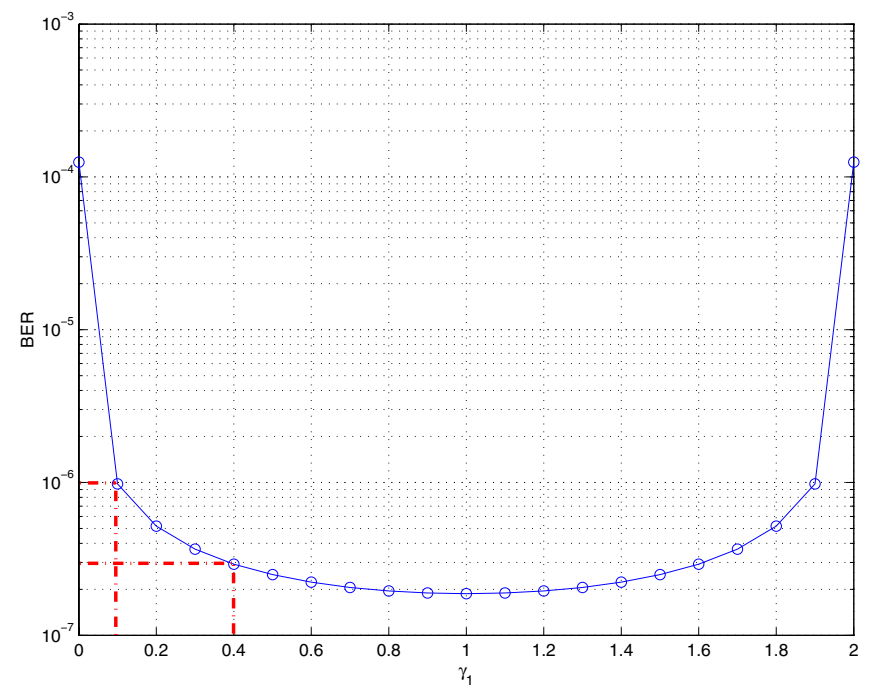

Figure. 5. BER as function of sub-channel gain $\gamma_{1}$.

performances can attain $10^{-5}$ for $S N R=30 \mathrm{~dB}$, for constant delay (A) and burst transmission (B) schemes. The FER per-

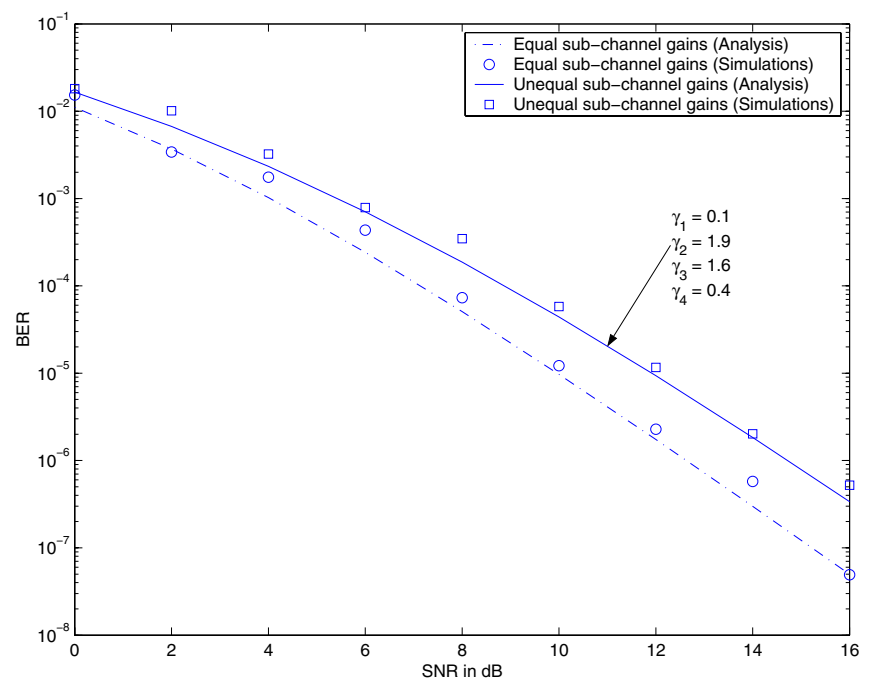

Figure. 6. BER performances of the asynchronous cooperative diversity system with channel and delay estimations for two receivers case, with equal and unequal sub-channel gains.

formances, as depicted in 8 , attain $4 \times 10^{-5}$ for $S N R=30 \mathrm{~dB}$.

\section{CONCLUSIONS}

In this paper, we proposed a precoding frame-based scheme with packet-wise encoding, which is implemented as $C P-$ training sequence. This scheme tolerates a relative delay as large as the cyclic prefix length, and the transmission delay and channel can be estimated efficiently from training sequences with a maximum likelihood (ML) method. The MSE of the channel estimation reaches the Cramer-Rao Bound and the BER performances show that we have a full diversity order even for the unbalanced channel gain cases.

\section{APPENDIX}

\subsection{CRB derivation}

The complex Fisher Information Matrix is :

$$
J(h)=\frac{1}{N_{0}}\left(\frac{\partial \mathbf{r}_{s}^{H}}{\partial h^{*}}\right)\left(\frac{\partial \mathbf{r}_{s}^{H}}{\partial h^{*}}\right)^{H}
$$

Where $\mathbf{r}_{s}=\mathbf{S}(\tau) \mathbf{h}$ is the signal part of $\mathbf{r}$. Therefore, we have :

$$
\begin{gathered}
\left(\frac{\partial \mathbf{r}_{s}^{H}}{\partial h^{*}}\right)=\frac{\partial \mathbf{h}^{H}(\mathbf{S}(\tau))^{H}}{\partial \mathbf{h}^{*}}=(\mathbf{S}(\tau))^{H} \\
\left(\frac{\partial \mathbf{r}_{s}^{H}}{\partial h^{*}}\right)^{H}=\mathbf{S}(\tau)
\end{gathered}
$$

\subsection{Derivation of the constants $K_{i}$}

It is proven here that

$$
K_{i}=\prod_{i^{\prime}=1, i^{\prime} \neq i}^{u} \frac{\gamma_{i}}{\gamma_{i}-\gamma_{i^{\prime}}}
$$


WPMC 2006 - San Diego, CA, USA

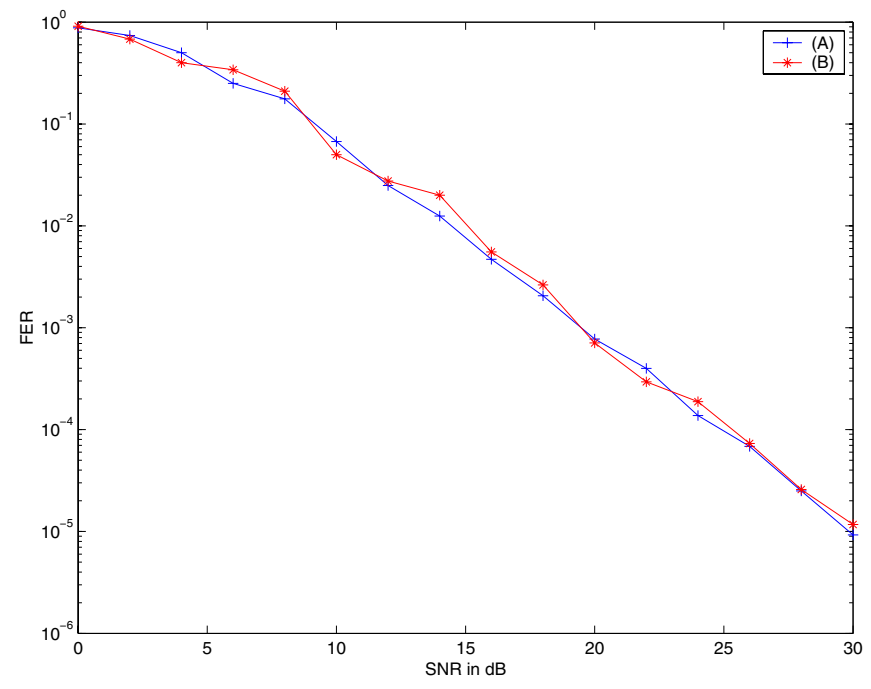

Figure. 7. FER performances of the asynchronous cooperative diversity system with channel and delay estimations, for one receiver case and equal sub-channel gains.

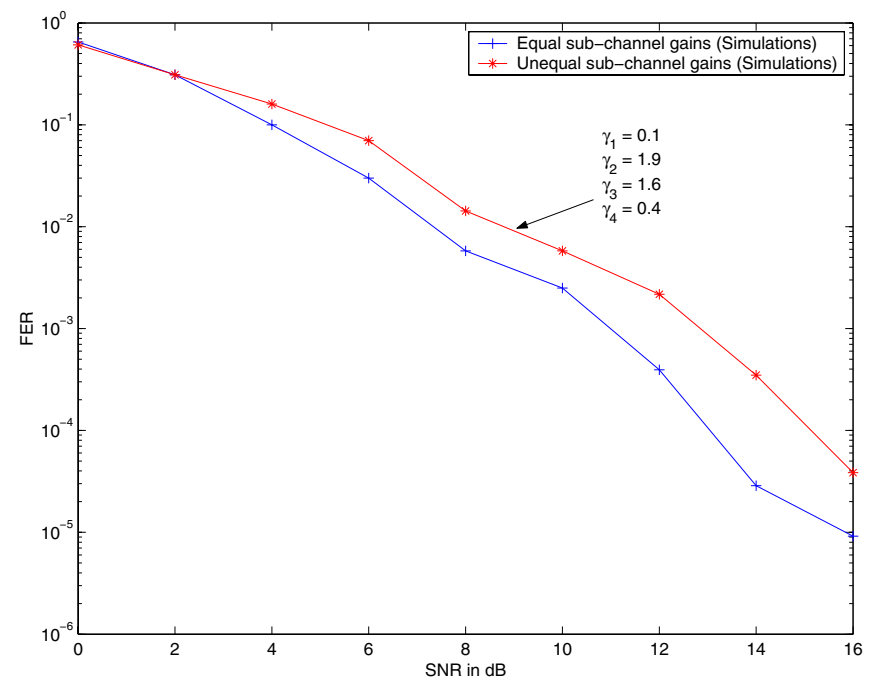

Figure. 8. FER performances of the asynchronous cooperative diversity system with channel and delay estimations for two receivers case, with equal and unequal sub-channel gains.
Without loss of generality, $K_{1}$ is derived here. The fractional expansion is equated to the product expression, i.e.

$$
\sum_{i^{\prime}=1}^{u} \frac{K_{i^{\prime}}}{1-\gamma_{i^{\prime}} s} \equiv \prod_{i^{\prime}=1}^{u} \frac{1}{1-\gamma_{i^{\prime}} s}
$$

To obtain $K_{1}$, (46) is multiplied by $\left(1-\gamma_{1} s\right)$ to yield

$$
\left(1-\gamma_{1} s\right) \quad \sum_{i^{\prime}=1}^{u} \frac{K_{i^{\prime}}}{1-\gamma_{i^{\prime}} s} \equiv\left(1-\gamma_{1} s\right) \quad \prod_{i^{\prime}=1}^{u} \frac{1}{1-\gamma_{i^{\prime}} s}
$$

after which $\mathrm{s}$ is set to $s=1 / \gamma_{1}$ to arrive at

$$
K_{1}=\prod_{i^{\prime}=2}^{u} \frac{1}{1-\frac{\gamma_{i^{\prime}}}{\gamma_{1}}}=\prod_{i^{\prime}=2}^{u} \frac{\gamma_{1}}{\gamma_{1}-\gamma_{i^{\prime}}}
$$

The same procedure is repeated for any $K_{i}$ to derive (45).

\section{REFERENCES}

[1] J. N. Laneman and G. W. Wornell, "Distributed spacetime-coded protocols for exploiting cooperative diversity in wireless networks," IEEE Trans. Inform. Theory, vol. 49, no. 10 , pp. 2415-2425, Oct. 2003.

[2] X. Li, "Space-time coded muli-transmission among distributed transmitters without perfect synchronization," IEEE Signal Processing Letters, vol. 11, no. 12, pp. 948-951, Dec. 2004.

[3] V. Tarokh, H. Jafarkhani, and A. Calderbank, "Space-time block codes from orthogonal design," IEEE Trans. Inform. Theory, vol. 45, no. 5, pp. 1456-1466, Jul. 1999.

[4] K. Tourki and L. Deneire, "'Precoding and Distributed STBC for Asynchronous Cooperative Diversity"," in The 8th International Symposium on Wireless Personal Multimedia Communications, Aalborg, Denmark, Sep. 2005.

[5] S. M. Alamouti, "A simple transmit diversity technique for wireless communications," IEEE Journal on Select Areas in Communcations, vol. 16, no. 8, pp. 1451-1458, Oct. 1998.

[6] C. Budianu and L. Tong, "'Packet Design For Communication Under Asynchronous Interference"," in Proc. of MILCOM, Boston, MA, Oct 2003.

[7] H. Shin and J. H. Lee, “'”Exact Symbol Error Probability of Orthogonal Space-Time Block Codes"," in Proc. of the IEEE Globecom., Taipei, Taiwan, Nov 2002. 